Erschienen in: Philosophy of Science ; 84 (2017), 4. - S. 737-760

https://dx.doi.org/10.1086/693874

\title{
The Volterra Principle Generalized
}

\author{
Tim RäZ*†
}

Michael Weisberg and Kenneth Reisman argue that the Volterra Principle can be derived from multiple predator-prey models and that, therefore, the Volterra Principle is a prime example for robustness analysis. In the current article, I give new results regarding the Volterra Principle, extending Weisberg's and Reisman's work, and I discuss the consequences of these results for robustness analysis. I argue that we do not end up with multiple, independent models but rather with one general model. I identify the kind of situation in which this generalization approach may occur, and I analyze the generalized Volterra Principle from an explanatory perspective.

1. Introduction. The Lotka-Volterra predator-prey model is historically important because it initiated mathematical modeling in biology. It is also philosophically important because it is a much-discussed case study in the debates on scientific modeling, explanation, idealization, and robustness analysis. One of the most important results of the Lotka-Volterra predator-prey model is the Volterra Principle. This principle, first formulated as the Third Law by Vito Volterra, is supposed to explain an empirical regularity about fishery statistics. However, the Lotka-Volterra predator-prey model is highly idealized, to the point that a realistic interpretation of the model is questionable. Does this mean that the Volterra Principle is questionable as well? Weisberg (2006b), and Weisberg and Reisman (2008) argue that this is not the case. They propose that the Volterra Principle can also be established from

Received September 2016; revised January 2017.

*To contact the author, please write to: FB Philosophie, Universität Konstanz, 78457 Konstanz, Germany; e-mail: tim.raez@gmail.com.

$\dagger$ I thank Claus Beisbart, Matthias Egg, Nick de Hoog, Mark Kot, Andrea Loettgers, Thomas Müller, Antje Rumberg, Tilman Sauer, Verena Wagner for comments on various drafts of the article and Dan Ward for proofreading. This work was supported by the Templeton World Charity Foundation through grant TWCF0078/AB46.

Philosophy of Science, 84 (October 2017) pp. 737-760. 0031-8248/2017/8404-0007\$10.00

Copyright 2017 by the Philosophy of Science Association. All rights reserved.

This work is licensed under a Creative Commons Attribution-NonCommercial 4.0 International License (CC BY-NC 4.0), which permits non-commercial reuse of the work with attribution. For commercial use, contact journalpermissions@press.uchicago.edu. 
other predator-prey models. According to Weisberg, the Volterra Principle is thus a prime example for the practice of robustness analysis.

In the current article, I present new results concerning the Volterra Principle, extending and refining Weisberg's and Reisman's work, and I discuss the consequences of these new results for robustness analysis. The results are in keeping with the goals of robustness analysis proposed by Weisberg. However, they deviate from the process of robustness analysis that Weisberg proposes. In particular, we do not end up with multiple independent models but rather with one general model. I identify the situations in which this generalization approach may apply. I analyze the generalized Volterra Principle from an explanatory perspective, and I show that my results exhibit several virtues of mathematical explanations. Finally, I discuss cases in which the generalization approach may not apply, and I propose that these are in fact cases of mathematical coincidences.

2. The Lotka-Volterra Predator-Prey Model. The Lotka-Volterra predatorprey model was originally proposed by Alfred Lotka and Vito Volterra. ${ }^{1}$ Formally, the model is a set of two coupled ordinary differential equations. These equations are intended to capture the interactions between a population of prey animals and a population of predators, which feed on the prey. ${ }^{2}$ The model can be written as follows:

$$
\begin{aligned}
& \dot{x}=r x-a x y . \\
& \dot{y}=b x y-m y .
\end{aligned}
$$

Here, $x$ is shorthand for $x(t)$, the size of the prey population at time $t ; y$ is shorthand for $y(t)$, the size of the predator population at time $t ; \dot{x}$ and $\dot{y}$ are the respective time derivatives. According to equation (1), the prey population $x$ grows at a constant positive rate $r$ in the absence of predators; in the presence of predators, the number of prey decreases proportionally to the number of predators, at a constant positive rate $a$. According to equation (2), the predator population $y$ decreases at a constant positive rate $m$ in the absence of prey; in the presence of prey, the predator population grows proportionally to the number of prey available, at a constant positive rate $b$. The system is coupled because both equations depend on both $x$ and $y$. Dynamically, this system exhibits undampened oscillations: if undisturbed, it will oscillate indefinitely on the same circular orbit in phase space (see fig. 1).

1. See Knuuttila and Loettgers (2016) for a discussion of the contrast between Lotka's and Volterra's approach to scientific modeling in general and to their eponymous model in particular.

2. Kot (2001) gives a detailed and accessible introduction to the mathematical aspects of the Lotka-Volterra model and predator-prey models in general. 


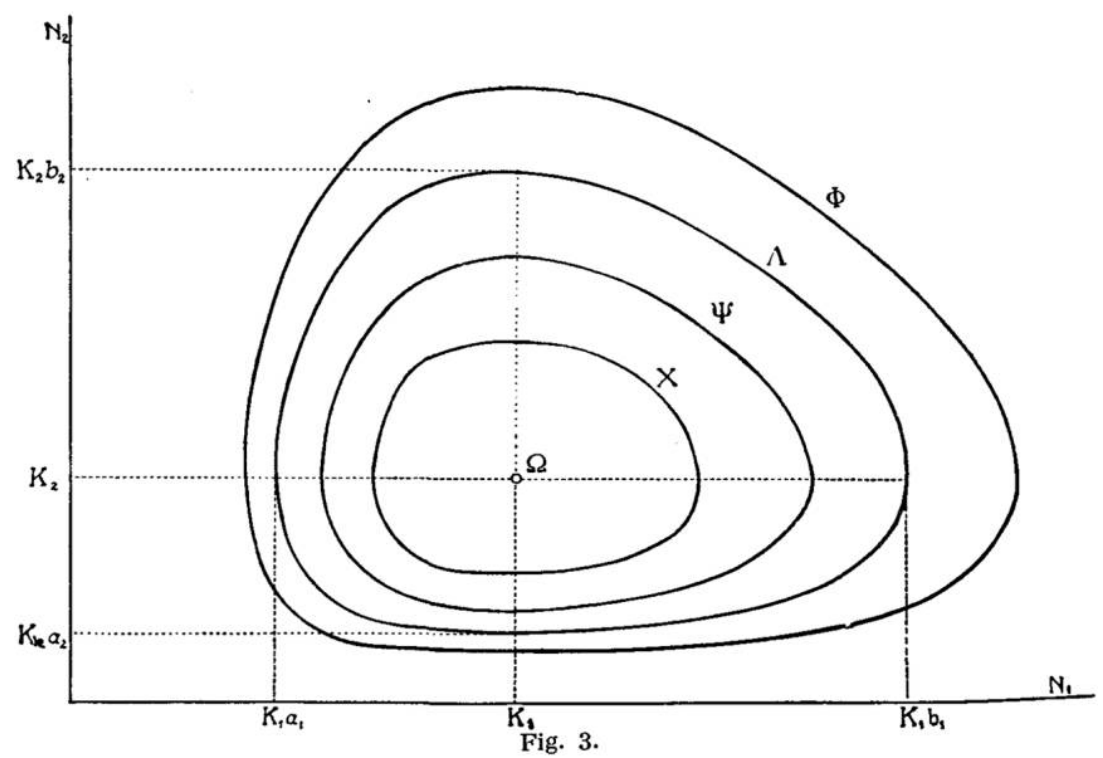

Figure 1. Periodic orbits in phase space: $N_{1}$ denotes the prey population; $N_{2}$ denotes the predator population. Orbits $X, \Psi, \Lambda, \Phi$ are examples of the exhaustive and disjunct orbits corresponding to systems with different initial conditions; $\Omega$ is the equilibrium state of all systems. If a system is not disturbed, it evolves on its orbit, running counterclockwise with a constant period, and it stays on its orbit; this is Volterra's First Law. Illustration from Volterra (1928, 14): by permission of Oxford University Press.

Volterra's motivation for formulating the predator-prey model was an empirical phenomenon in need of explanation. ${ }^{3}$ The explanandum concerns fishery statistics. During World War I, when fishing in the Adriatic was diminished, fishery statistics showed that the ratio between predators and prey fish shifted in favor of predators. After the war, when fishing returned to its prewar intensity, the ratio shifted back, and favored the prey. This prompted the question as to why this shift in proportions occurred. The explanation is provided by Volterra's Third Law, now called the Volterra Principle. The Volterra Principle states that, if we continuously remove a constant proportion of both the predator and prey populations, then the average number of predators will decrease relative to the average number of prey (see fig. 2). Volterra showed that this shift of averages can be derived from the predator-prey model. He thus provided a how-possibly explanation of the statistical phenomenon: the regularity holds in a very simple model of population dynamics. ${ }^{4}$

3. See Volterra (1928) for Volterra's own account of the story.

4. See, e.g., Forber (2010) and Scholl and Räz (2013) on the notion of how-possibly explanations. 


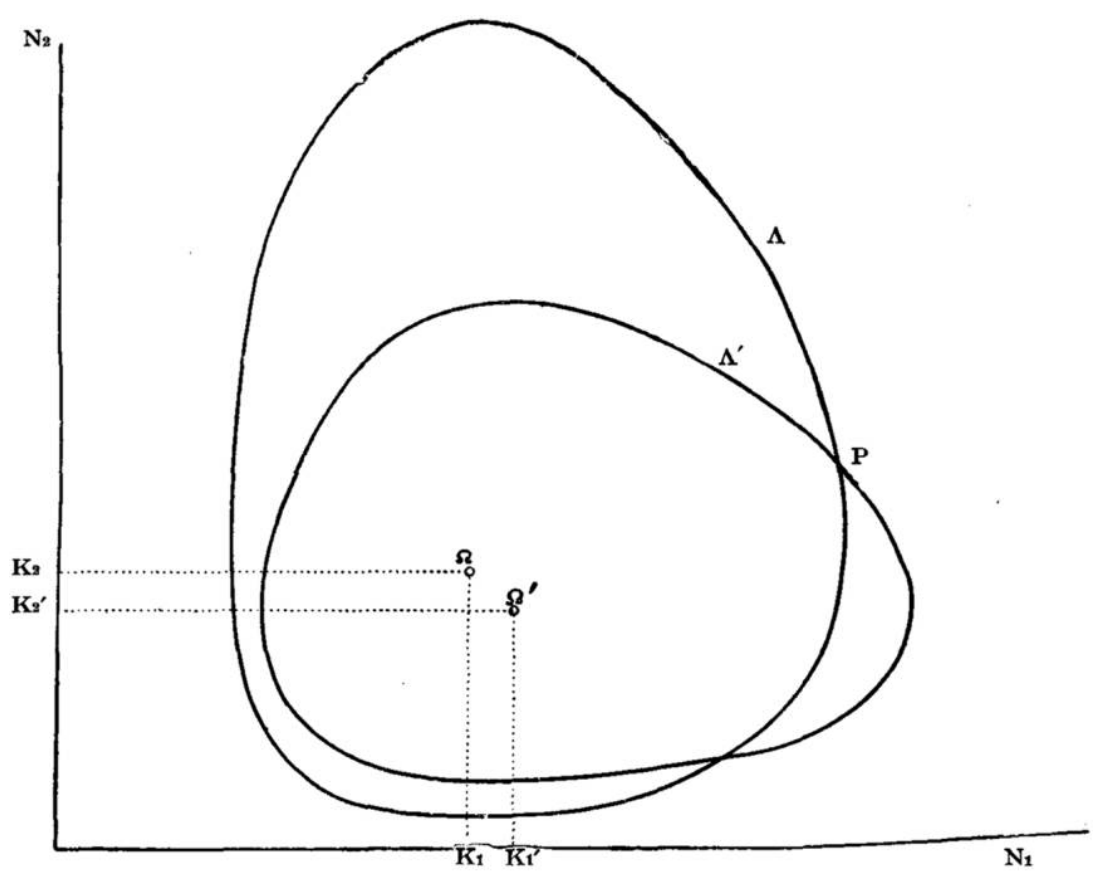

Fig. 6.

Figure 2. Population density of the predator-prey system with orbit $\Lambda$ at the equilibrium point $\Omega$ on average; this is Volterra's Second Law. If equations (1) and (2) of the system at point $P$ are changed, such that the predator death rate $m$ increases and the prey birth rate $r$ decreases, the system evolves on the new orbit $\Lambda^{\prime}$ with the equilibrium point $\Omega^{\prime}$. On average, system $\Lambda^{\prime}$ has a higher prey density $\left(K_{1}\right.$ to $\left.K_{1}^{\prime}\right)$ and a lower predator density $\left(K_{2}\right.$ to $\left.K_{2}^{\prime}\right)$. This is Volterra's Third Law. Before and after World War I, the system was on orbit $\Lambda^{\prime}$; during World War I, with greatly reduced fishing, the system was on orbit $\Lambda$. Illustration from Volterra $(1928,19)$ : by permission of Oxford University Press.

It has, however, been disputed that the Volterra Principle provides an actual explanation. Many mathematical population biologists today do not believe that the Lotka-Volterra model is a realistic representation of predatorprey interactions. The main mathematical problem with the model is that it is not structurally stable: if perturbations terms are added to the model equations, the model's qualitative behavior changes. ${ }^{5}$ Does this mean that the Volterra Principle is also doomed to fail? This question has been brought to philosophers' attention in two papers (Weisberg 2006b; Weisberg and Reisman 2008). Weisberg and Reisman argue that the Volterra Principle does not de-

5. See Brauer and Castillo-Chávez $(2001,180)$ for more on the notion of structural stability. 
pend on the specifics of the original predator-prey model. Rather, the Volterra Principle can be derived from a variety of models, which are more realistic than the original predator-prey model. In short, Weisberg and Reisman claim that the Volterra Principle is a "robust theorem." This makes the Volterra Principle a prime example for the practice of robustness analysis. In the next section, I give a brief introduction to robustness analysis, as well as to Weisberg and Reisman's account of the Volterra Principle.

\section{Robustness Analysis}

3.1. Early Contributions. Robustness analysis was first discussed as a scientific practice in a seminal paper by Levins (1966). ${ }^{6}$ Levins observes that population biology makes use of highly idealized models; that is, the models are based on assumptions that are, strictly speaking, false with respect to their target systems. How can we make sure, nonetheless, that results we derive from such models are still true with respect to their target systems and not mere artifacts of the idealizations of the models? Levins recommends robustness analysis as a remedy. The idea is to derive results from "several alternative models each with different simplifications but with a common biological assumption" (423). If this procedure is carried out successfully, the results are called "robust theorems." The slogan of robustness analysis is that "our truth is the intersection of independent lies" (423).

An early adopter of robustness analysis is Wimsatt (2012b; see also Wimsatt 2007). Wimsatt advocates a wide conception of robustness analysis, also dubbed "multiple determination," whereby the practice is not restricted to the derivation of phenomena from different models but rather encompasses, say, the detection of phenomena using different senses, or different experimental methods. Wimsatt emphasizes that it is important to determine the limitations of robustness results, given that robustness analysis involves, in part, understanding the scope and limitations of the robustness of a phenomenon.

An important critique of Levins's proposal has been formulated by Orzack and Sober (1993). Orzack and Sober point out that robustness analysis cannot play a confirmatory role. If all the models used in robustness analysis are idealized, then we do not know whether results derived from these models are true with respect to the target system. If we want to be certain, we would have to make sure that at least one of the models faithfully represents the target system. However, this is usually not the case. In short, independent lies are still lies. In reaction to Orzack's and Sober's objection, Levins (1993) argues that he did not intend robustness analysis to provide nonempirical 
confirmation; rather, robustness analysis is merely supposed to help in determining the scope and limits of a result.

3.2. Weisberg and Reisman. The most influential recent account of robustness analysis has been proposed by Weisberg (2006b). ${ }^{7}$ Weisberg aims to reconcile robustness analysis with the objections raised by Orzack and Sober. He proposes that robustness analysis does not confer full-blown confirmation but rather "low-level confirmation," that is, "confirmation of the fact that certain mathematical structures can adequately represent properties of target phenomena" (740). Weisberg characterizes robustness analysis as a four-step process. In the first step, one examines whether several distinct models share a property that is expected to be robust. In the second step, one tries to identify the "core structure" that is responsible for the robust property. The third step consists of an empirical interpretation of the mathematical structure. The fourth step consists of "stability analysis," with the purpose of finding out how sensitive the models are with respect to slight changes to their structure.

Weisberg uses the Volterra Principle as a case study to support his analysis. He argues that the Volterra Principle is robust, given that it can be obtained in different predator-prey models. ${ }^{8}$ Specifically, Weisberg claims, we can modify the structure of the original predator-prey model by adding density dependence and predator satiation and still obtain the Volterra Principle; see the appendix for a brief discussion of these models. Weisberg claims that the Volterra Principle can be shown to be valid for both of these models. Establishing that these two models satisfy the Volterra Principle corresponds to the first step of robustness analysis. Weisberg then argues that these models have a common structure: the growth rate of predators is mostly controlled by the growth rate of prey, and the growth rate of prey is mostly controlled by the death rate of predators. According to Weisberg, this common structure is responsible for the phenomenon that predators and prey are affected differently by continuous destruction of both species. The identification of the common structure concludes step 2 of robustness analysis.

Weisberg and Reisman (2008) push robustness analysis further, and they refine the results regarding the Volterra Principle. First, they distinguish three kinds of robustness. Parameter robustness investigates robustness with respect to a variation of parameters in a model. For example, the Volterra Principle is robust under variations of the parameters $a, b, r$, and $m$ in models (1)

7. The interest in Levins's paper, and robustness analysis, was renewed by Weisberg and Odenbaugh (2006). These papers initialized the "second wave" of robustness analysis. See Wimsatt (2012a) for a useful account of the development of robustness analysis.

8. Weisberg's ideas are based on suggestive remarks in Roughgarden (1979), a textbook in population ecology. 
and (2). Structural robustness encompasses the more general robustness across models with different (causal) structure (e.g., models with or without density dependence). ${ }^{9}$ Representational robustness encompasses models that are formulated in different frameworks (e.g., using continuous vs. discrete variables). Weisberg and Reisman then argue that the Volterra Principle is robust according to all three kinds of robustness. They provide a formulation of the Volterra Principle that modifies the "hypothesis" from Weisberg (2006b). The new formulation is based on the notions of negative coupling and biocide. A predator-prey system is negatively coupled "just in case increasing the abundance of predators decreases the abundance of prey and increasing the abundance of prey increases the abundance of predators" (Weisberg and Reisman 2008, 114). In a general biocide, the death rate of predators is increased and the birth rate of the prey is decreased..$^{10}$ The new formulation of the Volterra Principle reads as follows:

(VP) Ceteris paribus, if a two-species, predator-prey system is negatively coupled, then a general biocide will increase the abundance of the prey and decrease the abundance of predators. (114)

Weisberg and Reisman claim that this principle holds in general. However, they do not provide a conclusive answer as to the scope and limits of VP. Weisberg and Reisman analyze further models with respect to the Volterra Principle. In Weisberg (2006b), robustness analysis was restricted to parameter and structural robustness - the models are all formulated in the framework of coupled ordinary differential equations - while Weisberg and Reisman (2008) also includes an analysis of individual-based models (IBMs). These are finite models with discrete space and time, which can be taken to represent individual animals. In the IBMs considered by Weisberg and Reisman, the discrete transition rules are not deterministic but rather stochastic. The behavior of these models is investigated via simulations. Weisberg and Reisman argue that the Volterra Principle can be recovered in this framework, and they therefore conclude that the Volterra Principle is representationally robust.

3.3. After Weisberg and Reisman. The work by Weisberg and Reisman has renewed philosophers' interest in robustness analysis. Weisberg's account of robustness analysis and the notion of low-level confirmation have

9. Structural robustness is not to be confused with structural stability, a mathematical notion.

10. In the case of systems (1) and (2), $r$ is decreased and $m$ is increased. The notion is made precise in definition 1 , see sec. 4.1 below. 
received considerable attention. Calcott points out that a robust theorem is only as good as the set of models from which it has been derived and that this is not sufficient for confirmation. However, robustness can nevertheless be helpful by "narrowing the possibility space of candidate solutions" (Calcott 2011, 287). Houkes and Vaesen (2012) provide an in-depth analysis of Weisberg's work. The main lesson of their paper is that the evaluative role of robustness analysis concerning a set of models is only as good as the credibility of the model family from which the set of models is chosen. The idea of robustness analysis has also been applied to pure mathematics: Krömer (2012) discusses a potential role for robustness in the foundations of mathematics, and Corfield (2010) focuses on mathematical structures exhibiting a surprising "confluence" of structural properties. The idea of robustness analysis has also been applied to cases from biology in Knuuttila and Loettgers (2011) and to economics in Kuorikoski, Lehtinen, and Marchionni (2010).

Robustness analysis is also relevant to the issue of scientific and mathematical explanation. The idea that robustness analysis can enhance our understanding of a phenomenon was proposed early in the literature (e.g., by Wimsatt 2012b). However, the relevance of robustness analysis for explanation has not been systematically explored. A notable exception is a recent contribution by Schupbach (2016). Schupbach proposes that robustness analysis can be interpreted as an explanatory enterprise. The idea is that if we establish the robustness of a property, then we can rule out competing explanations of that property. Schupbach illustrates the idea with the Volterra Principle. Initially, we should not be confident that the Volterra Principle is generally valid because we have only derived it from the original predator-prey model, and the result could be due to the idealizations of this simple model. However, if we can establish the Volterra Principle for models with different idealizations, then, according to Schupbach, we can rule out certain competing explanations as to why the Volterra Principle holds, namely, explanations attributing this fact to idealizations that are not present in the new models.

3.4. Robustness Analysis and Mathematics. In the current article, I restrict my attention to one particular kind of robustness, namely, the case of robust theorems that are derived from multiple independent models. This is a special case of robustness, in comparison to the wider notion of robustness championed, for example, by Wimsatt. ${ }^{11}$ The focus of the discussion will be on the role of mathematics in robustness analysis. While it has been acknowledged in the philosophical discussion that mathematics has an important role to play in robustness analysis, a systematic exploration of this role is so far missing from the discussion. I strive to fill this lacuna here. I will argue that

11. See Calcott (2011) for a similar useful distinction between several kinds of robustness. 
mathematics can help us enhance our confidence in a robust result by showing that it does not depend on particular idealizations. However, this is not achieved by deriving the result from multiple independent models but rather by deriving the result from one general model. I will not argue that mathematics, or robustness analysis, can play a confirmatory role. My discussion of robustness analysis is based on scientific practice: in the next section, I present new results concerning the Volterra Principle.

\section{The Volterra Principle Generalized}

4.1. The Volterra Principle in a Gause-Type Model. Here, I generalize the results in Weisberg (2006b) regarding the Volterra Principle. More specifically, I prove that the Volterra Principle holds for a more general type of model, which was first proposed by Gause (1934). I use the following modification of a Gause-type model: ${ }^{12}$

$$
\begin{gathered}
\dot{x}=\operatorname{xrf}(x)-p(x) y, \\
\dot{y}=p(x) y-m y,
\end{gathered}
$$

where $x$ is prey abundance, $y$ is predator abundance, $r$ is the constant part of the prey growth rate, and $m$ is predator mortality. The prey growth rate function $f(x)$, which was missing in the original predator-prey model, is assumed to be differentiable for $x \geq 0$; additionally, it is assumed that $d f / d x=: f_{x} \leq 0$ and $f(0)=a>0$. The environment can have a carrying capacity, that is, a constant $K>0$ with $f(K)=0$. The functional response $p(x)$, which was assumed to be the identity function in the original predator-prey model, is assumed to be differentiable for $x \geq 0$; additionally, it is assumed that $d p /$ $d x=: p_{x}>0$ and $p(0)=0 .{ }^{13}$

The Volterra Principle states how the equilibrium of a dynamical system is affected by a change in the parameters of the system (see fig. 2). In order for this to be biologically meaningful, systems (3) and (4) need to have an equilibrium inside the first quadrant (i.e., an equilibrium with positive population variables). For the existence of such an equilibrium, we have to assume that there is an $x^{*}$ such that $p\left(x^{*}\right)=m$. For the formulation of the Volterra Principle, we additionally assume the existence of an $x^{\prime}$ with $p\left(x^{\prime}\right)=$ $m+\delta$ for a given $\delta>0$. Values $x^{*}$ and $x^{\prime}$ are unique because $p(x)$ is increas-

12. See Freedman (1980, chap. 4). In comparison to system (4.2) (Freedman 1980, 66), I use $r f(x)$ instead of $g(x)$ in eq. (3) and $p(x)$ instead of $c p(x)$ in eq. (4).

13. The numerical response, $p(x)$ in (4), is set equal to the functional response, $p(x)$ in (3). See Freedman (1980, chap. 4) for a further discussion of the significance of these assumptions. 
ing. If the system has a carrying capacity $K$, we have to assume that $x^{*}, x^{\prime}<K$ for the existence of an equilibrium.

The coordinates of the equilibrium in the first quadrant can be determined as follows: we set equations (3) and (4) equal to 0 and solve the resulting equations. Using the assumption that there is a unique $x^{*}$ with $p\left(x^{*}\right)=m$, we can deduce that the unique equilibrium has the following coordinates:

$$
\left(x^{*}, \frac{x^{*} r f\left(x^{*}\right)}{p\left(x^{*}\right)}\right) \text {. }
$$

The change in parameters, which leads to a change in equilibrium, can be interpreted as an intervention. The following definition makes this precise:

Definition 1. A Volterra Intervention in a predator-prey system is a decrease of $r$ by an amount $\varepsilon$, with $0<\varepsilon<r$, and an increase of $m$ by an amount $\delta>0$.

The assumption that $0<\varepsilon<r$ avoids a negative growth rate. And $0<\delta$ yields a new unique equilibrium in the positive quadrant after the Volterra Intervention. If we apply a Volterra Intervention to equilibrium (5), and use $p\left(x^{\prime}\right)=m+\delta$, we get a unique equilibrium with the following coordinates:

$$
\left(x^{\prime}, \frac{x^{\prime}(r-\varepsilon) f\left(x^{\prime}\right)}{p\left(x^{\prime}\right)}\right) .
$$

We are interested in the relation between equilibrium (5) and equilibrium (6); more specifically, how a Volterra Intervention affects the relative abundance of the populations:

Definition 2. A predator-prey system has the Volterra Property if a Volterra Intervention, applied to an equilibrium $\left(x^{*}, y^{*}\right)$, yields an equilibrium $\left(x^{\prime}, y^{\prime}\right)$ such that the equilibrium abundance of predators relative to prey (i.e., their ratio) decreases: $y^{*} / x^{*}>y^{\prime} / x^{\prime}$

If we examine figure 2, we see that the original Lotka-Volterra model has the Volterra Property: $K_{2} / K_{1}>K_{2}^{\prime} / K_{1}^{\prime}$ is true because $K_{2}>K_{2}^{\prime}$ and $K_{1}<K_{1}^{\prime}$. We are now in the position to formulate the following theorem:

Theorem 1. For Gause-type models (3) and (4), the Volterra Property holds true.

Proof. We have to show that the Volterra Property holds true for systems (3) and (4); that is, the ratio $y^{*} / x^{*}$ from equilibrium (5) is bigger than the ratio $y^{\prime} / x^{\prime}$ from equilibrium (6): 


$$
\frac{r f\left(x^{*}\right)}{p\left(x^{*}\right)}>\frac{(r-\varepsilon) f\left(x^{\prime}\right)}{p\left(x^{\prime}\right)} .
$$

This inequality can be rearranged to yield

$$
\frac{f\left(x^{*}\right)}{f\left(x^{\prime}\right)} \frac{p\left(x^{\prime}\right)}{p\left(x^{*}\right)}>1-\frac{\varepsilon}{r} .
$$

Because $p\left(x^{*}\right)=m, p\left(x^{\prime}\right)=m+\delta$, and because $p$ is strictly increasing, we know that $x^{\prime}>x^{*}$; that is, $x^{\prime}=x^{*}+\kappa$ for some $\kappa>0$. Replacing $x^{\prime}$ with $x^{*}+\kappa$, we see that (8) is equivalent to

$$
\frac{f\left(x^{*}\right)}{f\left(x^{*}+\kappa\right)} \frac{p\left(x^{*}+\kappa\right)}{p\left(x^{*}\right)}>1-\frac{\varepsilon}{r} .
$$

The fact that $f$ is a decreasing function implies that $f\left(x^{*}\right) / f\left(x^{*}+\kappa\right) \geq 1$, and the fact that $p$ is a strictly increasing function implies that $p\left(x^{*}+\kappa\right) /$ $p\left(x^{*}\right)>1$. This implies that the left-hand side of the inequality is bigger than 1 . The right-hand side, however, is smaller than 1 for $\varepsilon>0$. Therefore, the inequality holds true. QED

4.2. Stable Equilibria. Theorem 1 is a statement about how the equilibrium of a system changes under a Volterra Intervention. However, this is not sufficient for the result to be biologically meaningful. The existence of an equilibrium does not guarantee that the average abundance of a system coincides with the equilibrium. In the case of the original Lotka-Volterra model, this was guaranteed by Volterra's Second Law (see fig. 2). However, other predator-prey models show a variety of qualitatively different behaviors. For example, the so-called Samuelson model has an equilibrium that is stable or unstable depending on the choice of parameters. ${ }^{14}$ For some choices, the system spirals toward a stable equilibrium in the first quadrant. For other choices, the system spirals away from an unstable equilibrium. In the latter case, it is not relevant whether the Volterra Principle holds true because the equilibrium point is not where the system is on average. Rather, the system will show more and more violent fluctuations, such that the equilibrium shift predicted by the Volterra Principle is irrelevant. The same problem occurs in models with limit cycles. For example, the model with predator satiation examined in Weisberg $(2006 \mathrm{~b}, 736)$ satisfies the Volterra Principle according to theorem 1 . However, the system does not converge toward an equilibrium because the model exhibits limit cycles, and it is not clear how average abundance and equilibrium are related — a result analogous to Volterra's Second Law would be necessary to establish such a relation.

14. See Freedman $(1980,37)$ for a discussion of the Samuelson model. 
One way of making sure that the equilibrium is biologically relevant is to require that it is stable. ${ }^{15}$ Stability questions are standard in mathematical population ecology, and theorem 1 can be combined with an available stability criterion. An eigenvalue analysis of generalized Gause systems yields a criterion for the equilibrium's stability (see Freedman 1980, 72, condition [4.23]). This criterion implies that the relevant equilibrium of systems (3) and (4) is stable under the following condition: ${ }^{16}$

$$
H\left(x^{*}\right)=x^{*} f_{x}\left(x^{*}\right)+f\left(x^{*}\right)-\frac{x^{*} f\left(x^{*}\right) p_{x}\left(x^{*}\right)}{p\left(x^{*}\right)}<0 .
$$

This condition can be combined with theorem 1 as follows: if the equilibrium of systems (3) and (4), before and after the Volterra Intervention, is stable according to condition (10), then theorem 1 is certainly biologically meaningful.

4.3. The Limits of the Volterra Principle. An important advantage of theorem 1 is that condition (9) allows us to infer the limits of the Volterra Principle. In particular, condition (9) shows how the proof of the theorem depends on the assumptions about functions $f$ and $p$, and it also shows that these assumptions are minimal, in that if they are weakened further, the theorem does not hold. Here I will discuss two ways in which the Volterra Principle does not hold if the assumptions on $f$ and $p$ are weakened.

Possibility 1: Assume that $f_{x}>p_{x}>0$ in a range $x \in\left[x^{*}, x^{*}+\kappa\right]$; that is, $f$ is no longer decreasing but increasing faster than $p$. This implies that the left-hand side of (9) gets smaller than 1 and the inequality is violated for sufficiently small $\varepsilon$.

Possibility 2: Assume that $p_{x}<0$ (i.e., the functional response decreases). In this case, we have to be more careful because the theorem depends on $p_{x}>0$ in several places. First, we found that $p_{x}>0$ implies the uniqueness of the equilibrium. If $p_{x}<0$, there could be two equilibria or none. Second, we used $p_{x}>0$ to infer $x^{\prime}>x^{*}$. If $p_{x}<0$, this relation is inverted; this leads to a modification of condition (9):

$$
\frac{f\left(x^{*}\right)}{f\left(x^{*}-\kappa\right)} \frac{p\left(x^{*}-\kappa\right)}{p\left(x^{*}\right)}>1-\frac{\varepsilon}{r} .
$$

15. The relevant notion here is asymptotic stability, which means that systems close to equilibrium will eventually converge to that equilibrium. This is not to be confused with structural stability mentioned above. See Freedman (1980) and Kot (2001) for more on asymptotic stability.

16. The derivative of $f, p$ with respect to $x$, evaluated at $x^{*}$, is $f_{x}\left(x^{*}\right), p_{x}\left(x^{*}\right)$. 
If we assume that $p$ does not change sign, we need $f_{x}>\left|p_{x}\right|>0$ in the range $x \in\left[x^{*}-\kappa, x^{*}\right]$ in order to violate inequality (9') (i.e., $f$ has to increase faster than $p$ is decreasing). ${ }^{17}$

Are the conditions under which the Volterra Principle is violated biologically realistic? Consider, first, possibility $1: f_{x}>0$ is not realistic globally because it implies unbounded growth of prey in the absence of predators. However, $f_{x}>0$ seems reasonable in a limited range, in particular when the prey population is small and growth is not restricted externally. Turning to possibility 2, the case of a decreasing functional response $p$ has been discussed in the literature (see, e.g., Kot 2001, 140). According to Kot, socalled type IV functional responses can occur because of prey interference or prey toxicity. Thus, both possibilities potentially occur in situations that are biologically realistic.

It could be asked whether the assumptions of theorem 1, on the one hand, and the requirement of stable equilibria, on the other, are independent. Does the theorem exclude unstable equilibria, or can we find predator-prey models that violate the Volterra Principle and have stable equilibria at the same time? In order to answer this question, it is useful to reformulate the stability condition (10). If we assume that $f\left(x^{*}\right)$ is not $0,(10)$ is equivalent to

$$
\frac{f_{x}\left(x^{*}\right)}{f\left(x^{*}\right)}-\frac{p_{x}\left(x^{*}\right)}{p\left(x^{*}\right)}<-\frac{1}{x^{*}} .
$$

The expression on the left-hand side contains statements about the relative change in $f$ and $p$. The question now is whether there are cases in which condition (11) holds true while condition (9) is violated. Consider possibility 1 of violating (9); that is, $f_{x}>p_{x}>0$. This is compatible with stability (11) if $f\left(x^{*}\right)$ is sufficiently big, while $p\left(x^{*}\right)$ is sufficiently small. Turning to possibility 2 (i.e., $f_{x}>\left|p_{x}\right|>0$, and $p_{x}<0$ ), we see that we cannot get stability because the left-hand side of (11) will be positive, unless we admit negative functional responses. This shows that there are models with stable equilibria that violate the Volterra Principle, notably models that violate the Volterra Principle according to possibility 1 .

4.4. Further Generalizations. Can the above results be generalized further? Theorem 1 depends on the scope of systems (3) and (4). This type of model is dubbed "intermediate" by Freedman (1980). There is a lot of space for generalization between Gause-type models and fully general dynamical

17. If we use a function $q(x)$ instead of $p(x)$ in the predator eq. (4) in the model, there are more possibilities for violating the Volterra Principle - a decreasing $q$, combined with increasing $p$ and decreasing $f$, yields a violation of the Volterra Principle. However, it is unclear how realistic it is to assume that the functions $p$ and $q$ show a qualitatively different behavior. 
systems ${ }^{18}$ Here I want to mention a different kind of intermediate model that is important in population ecology. Systems (3) and (4) have so-called laissez-faire dynamics; that is, the functional response (which is also the numerical response) $p(x)$ does not depend on the predator abundance $y$. Some population ecologists believe that this is problematic because these models do not allow for nontrivial predator interference. ${ }^{19}$ To overcome the problems of laissez-faire dynamics, Arditi and Ginzburg (1989) have proposed so-called ratio-dependent predator-prey models in which the functional and numerical responses are functions of the ratio between predator and prey abundances. Ratio-dependent models have been hotly debated in population ecology in recent decades. ${ }^{20}$ It would be interesting to see whether the Volterra Principle can be extended to this type of model as well.

5. Weisberg's Volterra Principle Revisited. In this section, I discuss the ramifications of the results in section 4 for Weisberg and Reisman's work on the Volterra Principle, as far as the scientific and mathematical substance of their contribution is concerned. First, the fact that the Volterra Principle holds for models with density dependence and predator satiation, which was pointed out by Weisberg, is implied by theorem 1: one merely has to observe that these models are special cases of (3) and (4) (see the appendix). What is more, theorem 1 not only shows that these two models satisfy the Volterra Principle but also shows that this is due to the form of the growth function $f$ and of the functional and the numerical response $p$. I turn to the ramifications of these observations for robustness analysis below.

Second, the analysis of the scope of theorem 1, provided in section 4.3, shows that VP, the formulation of the Volterra Principle in Weisberg and Reisman (2008), is incorrect. This is particularly clear if we consider possibility 1 , according to which a violation of the Volterra Principle can occur if the growth function $f$ is increasing. However, $f$ is independent of negative coupling, which only concerns the interaction terms. Therefore, the Volterra Principle can be violated if there is negative coupling, and VP is wrong.

Third, the equilibrium of a predator-prey system need not necessarily be the (average) abundance of that system, as I noted in section 4.2 above. Weis-

18. A general approach to predator-prey models is via so-called Kolmogorov-type models (see, e.g., Freedman 1980, chap. 5). This approach was first proposed by Kolmogorov (1978). Kolmogorov-type models take general dynamical systems of two variables as a starting point and impose certain restrictions on these systems in order to obtain predatorprey relationships.

19. See Yodzis (1994) for a discussion of the notion of laissez-faire dynamics and reasons for taking predator interference into account.

20. See Arditi and Ginzburg (2012) for a book-length discussion of ratio-dependent models and Arditi and Ginzburg (2014) and Barraquand (2014) for discussion. 
berg and Reisman do not explicitly address this problem. I already pointed out that Weisberg $(2006 \mathrm{~b}, 736)$ proposes extending the Volterra Principle to a model with predator satiation (see the appendix). This model satisfies the Volterra Principle, according to theorem 1. However, the model exhibits limit cycles. An argument analogous to Volterra's Second Law would be necessary to show that the equilibrium of this system is relevant to the average abundance; without such an argument, the Volterra Principle may be irrelevant. There is no such argument in Weisberg (2006b). To be fair, Weisberg and Reisman $(2008,119)$ implicitly acknowledge that the stability of equilibria is relevant to the Volterra Principle. They compare the significance of equilibria, in the case of the original Lotka-Volterra predator-prey model, one the one hand, and in the case of the model with density dependent prey, on the other, and note that in the former case, the equilibrium is the average abundance, while in the latter case, the system will actually converge toward the equilibrium. They seem to endorse the system being at equilibrium (on average) as a necessary condition for the Volterra Principle's being biologically meaningful.

6. Generalization and Robustness Analysis. In this section, I discuss the upshot of the results in section 4 for robustness analysis, with a focus on Weisberg's (2006b) account of robustness analysis. In section 4, I introduced one general model, the Gause-type model, which encompasses all the different models from Weisberg (2006b). I then showed that the Gause-type model has the Volterra Property. This generalization shows that the models investigated by Weisberg are not really independent, but rather belong to the same type, and that they all satisfy the Volterra Principle because they are of this type.

Generalization and robustness analysis are similar in that they have the same goal. Both try to establish that a certain result, such as the Volterra Principle, does not depend on some of the idealizations of a particular model, such as the original Lotka-Volterra model. However, generalization achieves this goal on a path different from robustness analysis: it establishes that certain idealizations of the original model are not necessary and that weaker assumptions suffice because we can derive the same result from one more general model instead of using multiple independent models. Note that generalization does not get rid of all idealizations: The generalization of a result is only as good as the idealizations used in the general model. However, if we generalize a result, we get rid of some idealizations, that is, the assumptions dropped in the more general model. Furthermore, generalization can be superior to robustness analysis in that it may use less idealizations. In the current case, the Gause-type model is much more general than all the models used by Weisberg taken together, and thus it is preferable from the point of view of idealizations. 
There are further important differences between the two accounts. First of all, the scope of generalization is more limited than that of robustness analysis. Weisberg anticipates this point. In the following passage, he comments on a possible outcome of robustness analysis that is akin to generalization:

In straightforward cases, the common structure is literally the same mathematical structure in each model. In such cases one can isolate the common structure and, using mathematical analysis, verify the fact that the common structure gives rise to the robust property. However, such a procedure is not always possible because models may be developed in different mathematical frameworks or may represent a similar causal structure in different ways or at different levels of abstraction. Such cases are much harder to describe in general, relying as they do on the theorist's ability to judge relevantly similar structures. (Weisberg 2006b, 738)

The generalization approach pursued in section 4 is such a "straightforward case." The Volterra Principle turned out to be an actual theorem for the Gause-type model. I agree with Weisberg that such an outcome should not be expected in all cases - generalization has its limits. However, we can be more specific about the kinds of situations in which generalization may be applicable. I now turn to this task.

The generalization approach will usually not work if models are from different mathematical frameworks, as Weisberg notes. The results in section 4 concern models that are ordinary differential equations. Their robustness is thus restricted to this particular mathematical framework and does not extend to, say, IBMs, as discussed in Weisberg and Reisman (2008). Put differently, the generalization approach does not work in the case of representational robustness (i.e., when models belong to different mathematical frameworks): if two models belong to different frameworks, they may not share a common mathematical structure, and it is hard, if not impossible, to treat them in a formally unified manner. The generalization approach is usually restricted to cases of structural robustness (i.e., to models from the same mathematical framework).

If we want to apply the generalization approach, it is not sufficient that the models are formulated within the same mathematical framework. It can be impossible to find a common generalization of models, even if they are formulated in the same mathematical framework. For example, Volterra (1978) explores properties of population fluctuations; the third property, the "disturbance of averages," is the Volterra Property. In part 2, paragraph 5, Volterra generalizes the Volterra Principle to systems with $n$ species. Volterra notes that, in order for this to make sense, one has to restrict $n$-species models in several ways. For example, if one wants to be able to compare the abun- 
dances of predators and prey, one needs an absolute distinction between predators and prey. Cases in which one species is preyed on by a second species, while also being the predator of a third species, have to be excluded because it is not clear how the Volterra Principle should even be formulated in such cases. However, Volterra shows that, under certain technical assumptions, a generalized analogue of the Volterra Principle can be proven if the system contains an equal number of predator and prey species. This draws attention to the fact that the precise formulation of the property that is expected to be robust is important for generalization. In the cases examined by Volterra, the robust property is not the same for two species and for $n$-species systems. In the former case, the property is formulated in terms of the ratio of two species, whereas in the latter case, the property concerns the ratio of (equally many) predator and prey species. Thus, the generalization approach can be difficult to carry out, if the robust property is not the same in all cases and needs to be reformulated.

In the case of the models examined in Weisberg (2006b), which served as the starting point of generalization, we find that the robust property in question, the Volterra Property, is the same formal statement in all cases. This is not an assumption that is generally made in robustness analysis. Generally, a robust property can be characterized informally, and the statements are only required to be similar, or "biologically the same." A second commonality of the models examined in Weisberg (2006b) is that the derivations of the Volterra Property, while different, are similar.

In sum, I suggest that the generalization approach should be applied as a rule of thumb in the following situation: if a series of models, formulated in the same mathematical framework, yields identical formulations of a robust property, and the derivations proceed in a similar manner, we should try to identify one general model from which we can derive the robust property. In this kind of situation, robustness analysis serves as a heuristic strategy: the point of deriving the same result from a range of different models serves the ultimate goal of finding a general result by examining some instances first.

7. The Generalized Volterra Principle as an Explanation. The Volterra Principle was originally proposed as an explanation of an empirical regularity, as I pointed out in section 2 above. It is, therefore, natural to ask whether the results in section 4 are explanatorily significant. I discuss this issue in the current section. I argue that the results in section 4 exhibit several explanatory virtues that have been proposed in the debate on mathematical explanations.

Before I discuss the explanatory virtues, some clarifications are in order. The original explanandum is an empirical regularity observed in fishery statistics. These findings have been disputed on empirical and statistical grounds 
(e.g., by Pearson 1927). Of course, the facticity of the explanandum is necessary if we want to give a real explanation. ${ }^{21}$ Here I do not discuss empirical questions, but I rather assume that we are dealing with an actual empirical phenomenon, and I assess the theoretical virtues of the results in section 4 under this assumption. The explanation I discuss is that the Volterra Property in Gause-type models is explained by the fact that the growth rate function $f$ is decreasing, while the functional response $p$ is increasing.

One of the explanatory virtues of the above results consists in their generality, or unificatory power, and we can spell this out in terms of unification, as proposed by Kitcher (1989): if we compare Weisberg's results with those given here, we see that the explanation I propose is more unified and that it subsumes different, but similar, arguments given by Weisberg, under one argument scheme, in a natural way. Now, it has been argued in the literature on mathematical explanations that unification, and generality, cannot be all there is to mathematical explanation. ${ }^{22}$ I agree that while generality and unification do contribute to explanatory power, they are not all there is to it.

A second explanatory virtue of the results in section 4 is that they establish natural boundaries for the validity of the Volterra Principle. ${ }^{23}$ I showed that the Volterra Principle is not valid if the assumptions on the functions $f$ and $p$ are weakened any further. These properties are minimal, in that if the growth rate $f$ increases, the explanandum does not follow; similarly, the equilibrium may become unstable, if the functional response $p$ decreases. These properties are not only sufficient but also necessary for the Volterra Principle, against the background of Gause-type models. This fits nicely with a recent proposal by Pincock (2015), who requires conditions that are necessary as well as sufficient for "abstract explanations": a kind of mathematical explanation. Pincock's idea is that requiring the condition to be necessary as well as sufficient makes sure that there are no redundant assumptions in the explanans, in that if the assumptions are weakened, the explanation fails. This is exactly what we see here.

A third explanatory virtue of the Gause-type explanation emerges, if we analyze its relation to the other less general explanations. Volterra's explanation, based on the original predator-prey model, establishes that it is possible to account for the Volterra Property on the basis of systems of coupled differential equations. Weisberg showed that Volterra's result can be extended

21. Additionally, one should always compare the mechanism producing the empirical phenomenon with the model used in the explanation, in order to assess the explanation's adequacy.

22. See Hafner and Mancosu (2008) for a critical discussion of unification à la Kitcher in the context of mathematical explanation and Steiner (1978) for arguments against a characterization of explanatory power in terms of generality.

23. The importance of establishing the limits of a result to robustness analysis has been noted by Wimsatt (2012b). 
to other more realistic models. This establishes that the explanation is not an artifact of the idealizations of the original predator-prey model. What the explanation based on the Gause-type model achieves is the identification of the properties that are ultimately responsible for the explanandum, that is, the (mathematical) Volterra Property. By doing this, the Gause-type explanation not only accounts for the explanandum, but it also explains why the other explanations, proposed by Volterra and Weisberg respectively, worked in the first place: the growth function and the functional response of all these explanations have the right properties. The idea that explanatory relations of this kind, between different explanations, can be interpreted as an explanatory virtue has recently been proposed by Lange (2015). According to Lange, a mathematical result that answers questions that other results leave open is "deep" and has high explanatory power. This is a further important feature of the results presented here.

In sum, the results regarding the Volterra Principle presented above, have several explanatory virtues. They are general, they provide an explanans that is sufficient and necessary in the case of Gause-type models, and they are "deep," in explaining why previous explanations worked.

8. Robustness Analysis and Coincidence. While it is desirable to apply the generalization approach, there is no guarantee that it will always work. What if the generalization approach yields no result? In the current section, I address this question. I will argue that in cases in which generalization cannot be applied, robustness analysis and explanation pull in different directions. I restrict attention to cases in which generalization is a promising approach in principle, that is, cases in which the same mathematical statement is derived from multiple independent models within the same mathematical framework, as discussed in section 6 .

Consider, for a moment, the reasoning process of a theorist who wants to establish the robustness of a property, such as the Volterra Property. The theorist examines a range of different models within the same mathematical framework and succeeds in deriving the same property from different models. In this situation, the theorist can be confident that the property is in fact robust. However, the theorist will not stop there. She will also wonder whether the fact that she succeeded in deriving the result in such a way has a common explanation. In fact, there is a certain urgency to find such an explanation because this would account for the fact that multiple derivations are possible. If, however, no such explanation is to be had, what does this tell us? It may tell us that the existence of multiple derivations is a mere coincidence.

It could be questioned whether mathematical coincidences are possible at all, given that all mathematical truths are necessary. At the very least, the notion of a mathematical coincidence needs to be clarified. The notion has been discussed by Lange (2010). Lange gives the following example of a math- 
ematical coincidence: the thirteenth digit of the decimal expansion of both $e$ and $\pi$ is 9 . There is no reason, or common explanation, for this fact; at least, no such explanation is known. It is a mere coincidence. In a nutshell, Lange's account of mathematical coincidence is that two mathematical facts $F$ and $G$ are coincidences, if there is no common mathematical explanation of $F$ and $G$. If we want this definition to be nontrivial, we have to make some requirements on the "naturalness" that a common mathematical explanation would have to satisfy; otherwise, a mere conjunction of any two explanations would be sufficient. One of the main challenges is to spell out just what such a requirement of "naturalness" amounts to. I will not discuss this issue in the abstract but turn to the case at hand instead.

Lange's idea applies to the case of the Volterra Principle, as follows. The mathematical coincidence is that we can derive the Volterra Property from the original predator-prey model, as well as from both the model with density dependence and the model with predator satiation; all of this was established in Weisberg (2006b). This situation, the (apparent) coincidence, prompts the request for an explanation. The explanation is provided in section 4 above: the different models are special cases of the Gause-type model, from which the Volterra Property can be derived. ${ }^{24}$ Thus, in some cases, the process of robustness analysis can itself prompt a request for an explanation, and in a subset of these cases - including the generalized Volterra Principle - an explanation can be provided, and the apparent coincidence is explained away.

What about real coincidences? In these cases, we succeed in deriving the same property from multiple models, but there is no common explanation of the fact that these multiple derivations were possible. This fact is a mere coincidence. In this case, the following situation arises. Assume that we want to check whether some idealizations in a model $M$ are responsible for the explanation of a property $P$ or whether the explanation is independent of these idealizations. According to Schupbach (2016), we can do this using robustness analysis. Assume, further, that we construct two models, $M_{1}$ and $M_{2}$; that, in these two models, different idealizations from $M$ are removed; and that we succeed in deriving the property $P$ from $M_{1}$ and $M_{2}$. Assume, finally, that this is a mathematical coincidence. This means that there is no explanation as to why we succeeded in deriving the property $P$ from $M_{1}$ and $M_{2}$. If this is the case, we will not be able to construct a model $M^{\prime}$ that encompasses $M_{1}$ and $M_{2}$, and from which we can derive $P$, insofar as such a derivation constitutes an explanation telling us why the multiple indepen-

24. I am not suggesting here that all mathematical explanations have the form of generalizations or unification; I merely claim that the results in sec. 4 do in fact explain the (apparent) coincidence in Weisberg (2006b), i.e., that the Volterra Property can be derived from multiple models. 
dent derivations are possible. This, in turn, means that the different idealizations in $M$, which were removed in $M_{1}$ and $M_{2}$ individually, cannot be removed collectively by constructing one model $M^{\prime}$, such that $M^{\prime}$ would explain $P$. This is worrisome because having at least one of the idealizations seems to be essential to the derivation of the property $P$ : it is impossible to remove all the idealizations in the two models at the same time by constructing an explanatory model. Note that the explanatory goal of robustness analysis, according to Schupbach's proposal, can still be achieved to a certain degree. We can control for some idealizations in a model, by deriving a property from different models, thus ruling out these idealizations as explanations. However, some idealization cannot be removed from our explanation.

In sum, it has to be expected that generalization will not work in all cases. The interpretation of such cases as mathematical coincidences has interesting consequences, in particular the impossibility of finding a general explanatory model that removes all idealizations we would like to control for. This does not mean that generalization or mathematical coincidences speak against robustness analysis. Rather, we should interpret robustness analysis as a heuristic strategy that can lead to several possible outcomes. On the one hand, we may discover a general model that provides a unified explanation of a mathematical fact. On the other hand, we may find that a mathematical fact that can be derived from several independent models is merely an example of a mathematical coincidence. In the latter case, the outcome of robustness analysis is that an explanation is not to be had and that some idealizations are here to stay.

9. Conclusion. In the current article, I have shown that the Volterra Principle can be generalized for Gause-type models, extending results by Weisberg and Reisman. I have argued that this generalization has some of the virtues of mathematical explanations; in particular, the limits of the results establish necessary and sufficient conditions, and the results illuminate previous explanations. I have also proposed that cases in which the generalization approach is not applicable can be interpreted as mathematical coincidences.

The current article reveals a close connection between the goals of robustness analysis and those of purely mathematical work on theoretical models. Generalizing existing results is a very common practice in mathematics. However, there are other aspects of mathematics that should also be explored in the context of robustness analysis. First, more should be said about cases of representational robustness, where the robust results stem from different mathematical frameworks. These are cases of analogical reasoning in mathematics, the status of which is all but clear. Second, more complicated cases of structural robustness have scarcely been discussed so far. For example, a systematic study of "analogical generalizations," such as Volterra's general- 
izations of the Volterra Principle to $n$-species systems, would be worthwhile. Third, the results in the current article could also be further generalized to other kinds of predator-prey models.

\section{Appendix}

\section{Models with Density Dependence and Predator Satiation}

After normalizing parameters $a$ and $b$, the model with density dependent prey (Weisberg 2006b, eqq. [6] and [7]) can be written as

$$
\begin{gathered}
\dot{x}=x r\left(1-\frac{x}{K}\right)-x y . \\
\dot{y}=x y-m y .
\end{gathered}
$$

In this model, the first summand in equation (1) is modified such that the prey population does not grow indefinitely in the absence of predators, but rather the growth is limited by a maximal number of prey, the carrying capacity $K$. This model can be seen to be of Gause type by letting $f(x)=$ $1-(x / K)$ and $p(x)=x$.

The model with predator satiation (Weisberg 2006b, eqq. [9] and [10]), after normalizing parameters $a, b$, and $c$, can be written as

$$
\begin{gathered}
\dot{x}=\operatorname{xr}\left(1-\frac{x}{K}\right)-\left(1-e^{-x}\right) y . \\
\dot{y}=\left(1-e^{-x}\right) y-m y .
\end{gathered}
$$

This model takes into account that the rate at which predators capture prey may not be constant, as in the Lotka-Volterra model, but may rather decrease with increasing prey density. This model can be seen to be of Gause type, by choosing $f(x)=1-(x / K)$ and $p(x)=1-e^{-x}$.

\section{REFERENCES}

Arditi, R., and L. R. Ginzburg. 1989. "Coupling in Predator-Prey Dynamics: Ratio-Dependence.” Journal of Theoretical Biology 139 (3): 311-26.

- 2012. How Species Interact: Altering the Standard View on Trophic Ecology. New York: Oxford University Press.

— 2014. "Improving Communications between Theoretical Ecologists, Mathematical Ecologists, and Ecological Modelers: Response to the Critique of Our Book How Species Interact." Theoretical Ecology 7:21-22.

Barraquand, F. 2014. "Functional Responses and Predator-Prey Models: A Critique of Ratio Dependence." Theoretical Ecology 7:3-20. 
Brauer, F., and C. Castillo-Chávez. 2001. Mathematical Models in Population Biology and Epidemiology. Texts in Applied Mathematics 40. New York: Springer.

Calcott, B. 2011. "Wimsatt and the Robustness Family: Review of Wimsatt's Re-engineering Philosophy for Limited Beings.” Biology and Philosophy 26:281-93.

Corfield, D. 2010. “Understanding the Infinite I: Niceness, Robustness, and Realism." Philosophia Mathematica 18 (3): 253-75.

Forber, P. 2010. "Confirmation and Explaining How Possible." Studies in History and Philosophy of Science C 41:32-40.

Freedman, H. I. 1980. Deterministic Mathematical Models in Population Ecology. Pure and Applied Mathematics 57. New York: Dekker.

Gause, G. F. 1934. The Struggle for Existence. Baltimore: Williams \& Wilkins.

Hafner, J., and P. Mancosu. 2008. "Beyond Unification.” In The Philosophy of Mathematical Practice, ed. P. Mancosu, 151-78. Oxford: Oxford University Press.

Houkes, W., and K. Vaesen. 2012. "Robust! Handle with Care.” Philosophy of Science 79:1-20.

Kitcher, P. 1989. "Explanatory Unification and the Causal Structure of the World." In Scientific Explanation, ed. P. Kitcher and W. C. Salmon, 410-505. Minnesota Studies in the Philosophy of Science 13. Minneapolis: University of Minnesota Press.

Knuuttila, T., and A. Loettgers. 2011. "Causal Isolation Robustness Analysis: The Combinatorial Strategy of Circadian Clock Research.” Biology and Philosophy 26:773-91.

— 2016. "Modelling as Indirect Representation? The Lotka-Volterra Model Revisited." British Journal for the Philosophy of Science. doi:10.1093/bjps/axv055.

Kolmogorov, A. 1978. "On Volterra's Theory of the Struggle for Existence." In The Golden Age of Theoretical Ecology, 1923-1940, ed. F. M. Scudo and J. R. Ziegler, 287-92. Lecture Notes in Biomathematics 22. Berlin: Springer. Originally published as "Sulla theoria di Volterra della lotta per l'esistenza" (1936).

Kot, M. 2001. Elements of Mathematical Ecology. Cambridge: Cambridge University Press.

Krömer, R. 2012. "Are We Still Babylonians? The Structure of the Foundations of Mathematics from a Wimsattian Perspective.” In Characterizing the Robustness of Science, ed. L. Soler, chap. 8, 189-206. Boston Studies in the Philosophy of Science. New York: Springer.

Kuorikoski, J., A. Lehtinen, and C. Marchionni. 2010. "Economic Modelling as Robustness Analysis." British Journal for the Philosophy of Science 61:541-67.

Lange, M. 2010. "What Are Mathematical Coincidences (and Why Does It Matter)?" Mind 119:307-40.

. 2015. "Depth and Explanation in Mathematics." Philosophia Mathematica 23 (2): 196-214.

Levins, R. 1966. "The Strategy of Model Building in Population Biology." American Scientist 54 (4): 421-31.

— 1993. "A Response to Orzack and Sober: Formal Analysis and the Fluidity of Science." Quarterly Review of Biology 68 (4): 547-55.

Odenbaugh, J. 2006. "The Strategy of 'the Strategy of Model Building in Population Biology.'” Biology and Philosophy 21:607-21.

Orzack, S., and E. Sober. 1993. "A Critical Assessment of Levins's the Strategy of Model Building in Population Biology, 1966." Quarterly Review of Biology 68 (4): 533-46.

Pearson, E. 1927. "The Application of the Theory of Differential Equations to the Solution of Problems Connected with the Interdependence of Species." Biometrika 1 (19): 216-22.

Pincock, C. 2015. "Abstract Explanations in Science.” British Journal for the Philosophy of Science 66 (4): 857-82.

Roughgarden, J. 1979. Theory of Population Genetics and Evolutionary Ecology: An Introduction. New York: Macmillan.

Scholl, R., and T. Räz. 2013. "Modeling Causal Structures.” European Journal for Philosophy of Science 3 (1): 115-32.

Schupbach, J. N. 2016. "Robustness Analysis as Explanatory Reasoning." British Journal for the Philosophy of Science. doi:10.1093/bjps/axw008.

Steiner, M. 1978. "Mathematical Explanation.” Philosophical Studies 34 (2): 135-51.

Volterra, V. 1928. "Variations and Fluctuations of the Number of Individuals in Animal Species Living Together." ICES Journal of Marine Science 3 (1): 3-51.

- 1978. "Variations and Fluctuations in the Numbers of Coexisting Animal Species." In The Golden Age of Theoretical Ecology, 1923-1940, ed. F. M. Scudo and J. R. Ziegler, 65-236. 
Lecture Notes in Biomathematics 22. Berlin: Springer. Originally published as "Variazioni e fluttuazioni del numero d'individui in specie animali conviventi" (1927).

Weisberg, M. 2006a. "Forty Years of 'the Strategy': Levins on Model Building and Idealization." Biology and Philosophy 21:623-45.

. 2006b. "Robustness Analysis." Philosophy of Science 73 (5): 730-42.

Weisberg, M., and K. Reisman. 2008. "The Robust Volterra Principle.” Philosophy of Science 75 (1): 106-31.

Wimsatt, W. C. 2007. Re-engineering Philosophy for Limited Beings. Cambridge, MA: Harvard University Press.

- 2012a. "Robustness: Material, and Inferential, in the Natural and Human Sciences." In Characterizing the Robustness of Science, ed. L. Soler, chap. 3, 89-104. Boston Studies in the Philosophy of Science. New York: Springer. Originally published in M. Brewer and B. Collins, eds., Scientific Inquiry in the Social Sciences (San Francisco: Jossev-Bass, 1981).

2012b. "Robustness, Reliability, and Overdetermination. In Characterizing the Robustness of Science, ed. L. Soler, chap. 2, 61-87. Boston Studies in the Philosophy of Science. New York: Springer. Originally published in M. Brewer and B. Collins, eds., Scientific Inquiry in the Social Sciences (San Francisco: Jossev-Bass, 1981).

Yodzis, P. 1994. "Predator-Prey Theory and Management of Multispecies Fisheries." Ecological Applications 4 (1): 51-58. 